\title{
Inter-patch movement in an experimental system: the effects of life history and the environment
}

\author{
Daniel O'Sullivan, Tim G. Benton and Tom C. Cameron \\ D. O'Sullivan, T. G. Benton and T. C. Cameron, (tcameron@essex.ac.uk), Inst. of Integrative and Comparative Biology, Univ. of Leeds, Leeds, \\ UK. TCC also at: Ecology and Environmental Sciences, Umeå University, SE-901 87 Umeå, Sweden.
}

\begin{abstract}
An important process for the persistence of populations subjected to habitat loss and fragmentation is the dispersal of individuals between habitat patches. Dispersal involves emigration from a habitat patch, movement between patches through the surrounding landscape, and immigration into a new suitable habitat patch. Both landscape and physical condition of the disperser are known to influence dispersal ability, although disentangling these effects can often be difficult in the wild. In one of the first studies of its kind, we used an invertebrate model system to investigate how dispersal success is affected by the interaction between the habitat condition, as determined by food availability, and life history characteristics (which are also influenced by food availability). Dispersal of juvenile and adult mites (male and female) from either high food or low food natal patches were tested separately in connected three patch systems where the intervening habitat patches were suitable (food supplied) or unsuitable (no food supplied). We found that dispersal success was reduced when low food habitat patches were coupled to colonising patches via unsuitable intervening patches. Larger body size was shown to be a good predictor of dispersal success, particularly when the intervening landscape is unsuitable. Our results suggest that there is an interaction between habitat fragmentation and habitat suitability in determining dispersal success: if patches degrade in suitability and this affects the ability to disperse successfully then the effective connectance across landscapes may be lowered. Understanding these consequences will be important in informing our understanding of how species, and the communities in which they are embedded, may potentially respond to habitat fragmentation.
\end{abstract}

Many species face an enhanced risk of extinction due to habitat loss and fragmentation (Heidinger et al. 2010), where increased extinction risk may arise from reduced population size and connectivity such that genetic variation and fitness is lost (Frankham et al. 2002). An important determinant for the survival of these populations is the dispersal of individuals between habitat patches (Thomas 2000). Individual benefits from dispersal can include avoidance of inbreeding and reduced mate and resource competition, particularly from kin (Bowler and Benton 2005). Dispersal involves three different conceptual stages: emigration from a habitat patch, movement between patches through the surrounding landscape, and immigration into a new suitable habitat patch (Bowler and Benton 2005). Successful dispersal therefore is dependent on the ability of a species to complete all three of these stages (Heidinger et al. 2010). Here we introduce a study that considers the role of environmentally determined individual condition, stage of development (i.e. juvenile vs adult) and the effective connectance between suitable habitat patches via

This is an open access article under the terms of the Creative Commons Attribution License, which permits use, distribution and reproduction in any medium, provided the original work is properly cited. manipulating patch suitability on the ability to successfully disperse from a deteriorating local environment.

Body mass and physical condition are important determinants of dispersal ability, with studies often showing higher dispersal rates in individuals of larger body size and better body condition (Barbraud et al. 2003, Gyllenberg et al. 2008, Warner and Shine 2008, Heidinger et al. 2010, Marini and Martins 2010, Clobert et al. 2012). This has been attributed to the potentially high costs associated with dispersal due to vulnerability of predation, difficulties in locating food, and aggression from individuals already inhabiting the new habitat patch, in addition to stored reserves allowing continued movement, and larger size increasing dispersal speed (Gaines and McClenaghan 1980, van Vuren and Armitage 1994, Waser et al. 1994, Bonte et al. 2012). Dispersal can be gender biased, being linked to differences in body condition and size, and associated with the reproductive roles of each sex (Dobson 1982, Greenwood and Harvey 1982, Olsson and Shine 2003, Benton and Bowler 2012). In general, we may expect differences in dispersal propensity and success to depend on developmental stage, body condition and the interaction between the two. For example, if dispersal is dependent on body size, we may expect juveniles to have 
lower dispersal success, but they may simultaneously have a higher dispersal propensity: smaller individuals can be more motivated to disperse due to competition (McCauley 2010, Einum et al. 2012). However, it has been shown for juvenile male red deer that body condition has no effect on dispersal where the cost of dispersal is low in terms of energy expenditure and survival (Loe et al. 2010). Unlike phenotype-dependent dispersal that occurs frequently in invertebrates, reptiles and small mammals (Clobert et al. 2001), the lack of effect of body mass on dispersal in red deer is thought to be due to individuals using migration corridors that often consist of normal feeding habitat combined with low rates of natural predation where subsequently the costs of dispersal are relatively small. This therefore implies a three-way interaction between age/ stage, condition and the 'effective connectance' between suitable habitat patches in determining movement rates across a landscape. The effective connectance will be a function of both the characteristics of the matrix through which individuals move and the suitability of habitat patches. If the matrix is not costly to move through and if every habitat patch is suitable the landscape is more effectively connected than if the matrix is harsh and some habitat patches are unsuitable (Bodasing et al. 2002, Barbraud et al. 2003, Telfer et al. 2003, Bowler and Benton 2009b).

It is difficult to predict how species will respond to habitat fragmentation as dispersal (propensity and success) has complex causation (Bowler and Benton 2005, Benton and Bowler 2012, Bonte et al. 2012): ecologically similar species therefore may respond differently. Further empirical studies on dispersal are therefore important. Two recent studies have used a laboratory-based model system, the soil mite Sancassania berlesei, to investigate how inter-patch distance, age, sex and food availability during an individual's developmental period effects dispersal propensity in a two-patch system (Bowler and Benton 2009a, b). Using the soil mite and a three-patch connected system, we aimed to investigate the interaction between natal patch conditions (high or low food), individual stage and condition, and the effective connectedness of the system (in terms of intervening patch suitability) in determining dispersal success. The current dispersal literature, including a large proportion using models to predict dispersal under particular parameters, focuses on the size or just presence of stepping stone habitat patches and not their suitability with relation to their effect on dispersal. To our knowledge our study is the first to use a model system to investigate not only the effects of stepping stone presence on dispersal but also how the suitability of the stepping stone patch can affect dispersal of individuals that vary in their condition due to differing natal patch food availbility.

\section{Methods}

\section{Study system}

A three patch laboratory system was used to investigate the dispersal of the soil mite Sancassania berlesei. A patch consisted of a glass test tube $(50 \mathrm{~mm}$ tall and $25 \mathrm{~mm}$ in diameter) with a plaster-of-paris substrate. Each test tube was connected using silicone tubing that acted as movement corridors. Previous experiments have shown that mites disperse between population patches (Bowler 2006, Bowler and Benton 2009a). Mites for the experiment were taken from a stock population created using large numbers of mites from two stock cultures that were set up in 2005. The stock population was fed ad libitum. This was to minimise any maternal effects that may have influenced the results, as we were most interested in the influence of environmental effects as a consequence of our controlled environmental manipulations. Unlit incubators maintained at $24^{\circ} \mathrm{C}$ were used to house the stock and experimental populations. Individual mites can take 7-8 days to mature from the egg stage and adults tend to live between ca 10-20 days under high food conditions, however the life history is resource dependent (Plaistow et al. 2004).

\section{Experiment}

This experiment explored the impacts of natal patch food provisioning (high or low food) and intervening patch suitability (food present or absent) on dispersal success in old juvenile (protonymph), and young adult male and female soil mites. These age groups were selected as they were shown to have the highest propensity to disperse (Bowler and Benton 2009b). Natal populations were set up in a total of 111 tubes, each with 30 pairs of adults and roughly 50 juveniles from the stock population. A total of 81 tubes were given a low food level of two small granules of yeast twice a week (weight of one granule: $0.49 \mathrm{mg} \pm$ 0.11 , mean $\pm S D, n=52$ ), and 30 tubes at high food level of three large granules of yeast daily (weight of one granule: $1.57 \mathrm{mg} \pm 0.15$, mean $\pm S D, n=52$ ). Populations were fed on low and high food regimes to create different levels of natal patch provisioning that would produce individuals with contrasting life histories due to resource competition (Plaistow et al. 2004). Each experimental treatment was split into two blocks a week apart to make the experiment more manageable, with the populations set up on day 11 and 18 from when the stock population was started. Population tubes were reared for 21 days (roughly 1-2 generations). This was to allow natal patch provisioning/competition effects to build up in the population while being too short a time period to give rise to evolution in the population that may alter an individual's response to these effects (Cameron et al. 2013). In order to ensure young adults were obtained, all adults were culled on day 18 in the low food populations and on day 20 in the high food populations. Thirty randomly selected individuals, chosen from the low or high food natal population tubes, of juveniles, males, or females were placed into the source patch of a three-patch experimental system. Each of the four treatments (high food-food present, lowpresent, high-absent, low-absent) was replicated five times per life history stage resulting in a total of 60 experiments.

In each experiment a source patch was connected to one intervening habitat patch, which itself was connected to a final colonization patch, with the use of two $15 \mathrm{~cm}$ silicone tubes (Fig. 1). No food was supplied in the source patch. The intervening patch contained either a small disk of food ( $2 \mu \mathrm{l}$ drop of a $10 \mathrm{ml}$ solution containing $0.5 \mathrm{~g}$ 


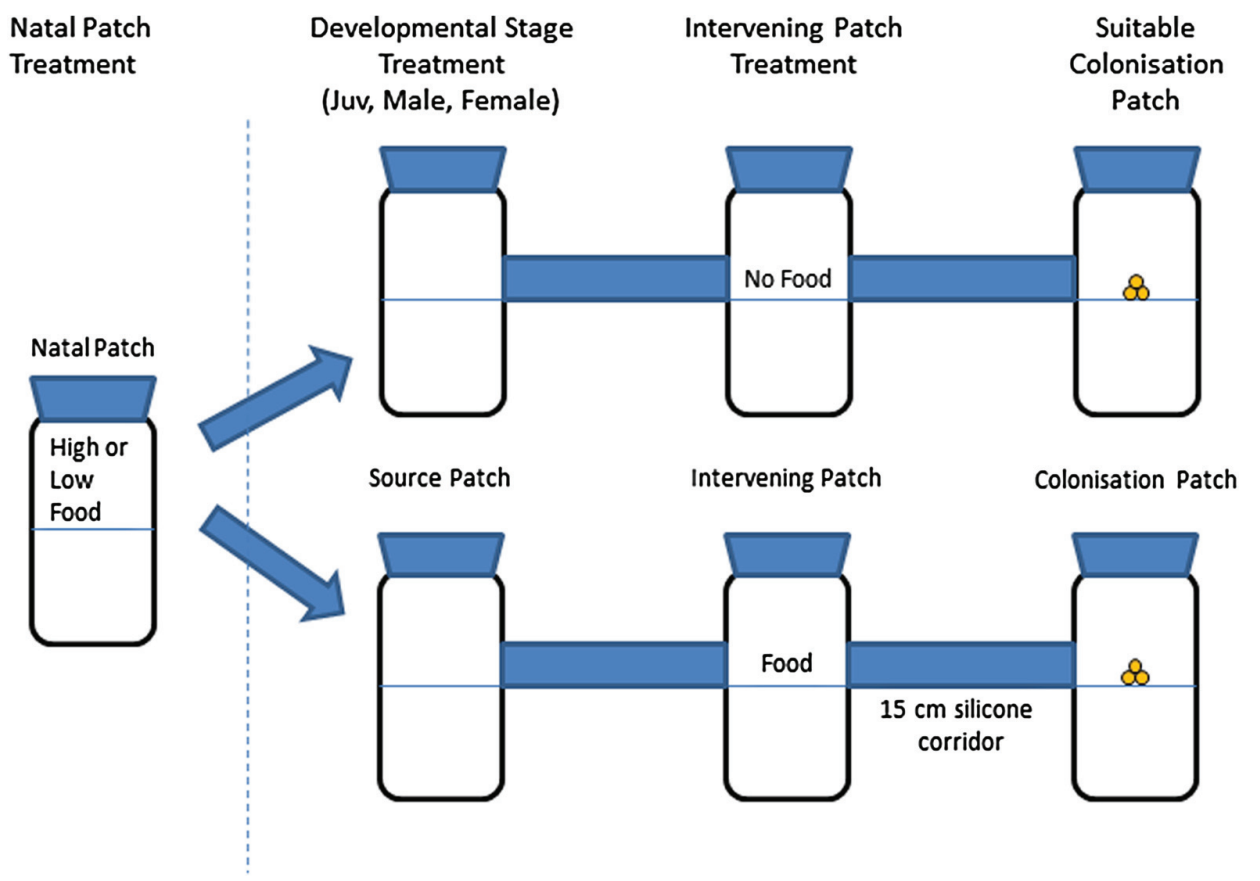

Figure 1. Schematic diagram showing the layout of the three-patch experimental system. A patch consisted of a glass test tube with a plaster-of-paris substrate. Juvenile, male or female mites from either high resource (high food) or low resource (low food) natal patch environments were tested on their ability/success to disperse from an unsuitable to a suitable patch dependent on their natal patch environment, developmental stage and suitability of the intervening patch habitat.

dissolved yeast), which was added daily, or did not (food present or absent). The colonization patch always contained three granules of yeast equivalent to those used for the high food populations. One drop of water was added daily to each patch. The silicone tubes are costly habitats to traverse as the low humidity increases mortality, whereas the intervening patch, with its damp plaster-of-paris base, is a more suitable habitat irrespective of the food supply. Juveniles, males, and females were tested in separate systems to determine specific impacts on their ability to disperse successfully. Counts were made daily of immigrants into the colonization patch, and those that were found in this patch were removed to ensure individuals were not recounted. Each three-patch experimental system ran for seven days, with counts taken during the six days following the initial set-up. A previous experiment investigating soil mite dispersal in a similar two-patch experimental system showed most ( ${ }^{\circ} 90 \%$ in a two-patch system) dispersal of juveniles and adults occurred within the first seven days of being placed into the source patch of the system (Bowler 2006). Juveniles, females and males were noted in a pilot experiment to be capable of dispersing from the source patch to the colonisation patch within $30 \mathrm{~min}$ to one hour. Thus a dispersal event within the experimental system takes up a fraction of an individual's lifespan considering adults can live between $10-20$ days and juveniles up to 50 days. Therefore, in this study, all individuals that had not yet reached the colonisation patch by day 7 were assumed to have failed.

Successful emigration from the source patch is difficult to estimate as we cannot differentiate between individual mites. Therefore mites could leave the source patch and return. However, from our daily counts the average emigration rate from source patches was $92 \%$ across all treatments (range 65-100\%). Emigration rates were not analysed further. Differentiating between dispersal failure and mortality is extremely challenging due to the difficulty in locating cadavers, especially if mites died during dispersal through the silicone tubes as they can be difficult to see. Dispersal mortality could be estimated assuming that all individuals not recovered from the system had died, e.g. mortality $=$ total-success-failed/total. However, the estimated dispersal mortality was highly negatively correlated with dispersal success $(-0.87)$ across all treatments. Due to the fixed density of mites in each experiment this is expected. To investigate the effects of natal patch environment on individual phenotype and whether individuals that dispersed successfully and those that failed differed in size, photographs of eggs, protonymphs and mature adults were taken from low and high food populations as-well as the three-patch experimental systems at $40 \times$ magnification using a stereomicroscope with 2MPEG camera unit attached. Measurements were then made of the longest length of eggs and individuals (from the tip of the hypostome to the tip of the idiosoma) using 'Eclipse Net' software.

\section{Data analysis}

We define dispersal success as movement from the source, via an intermediate patch to the colonization patch. We used the data collected to explore patterns in dispersal success between individuals from high or low natal environments in source patches as they disperse across habitats of different suitability. Dispersal success into a colonization patch, is the fraction of the original cohort that were found (and then removed) in this patch over the course of the experiment. The length measurements taken of individuals 
were used to test for size variation between the two differing natal patch qualities (high or low food) and also between individuals that dispersed successfully or failed. The results were used to explain any differences in an individual's ability to successfully disperse.

Data points were grouped according to temporal block (block 1 or 2). Generalised linear mixed models (glmer) with binomial error distributions were used to incorporate block as a random effect to account for replicates nested within each block and the associated sampling error. This was to ensure that any noise as a result of the grouping in the data from our block design would not hide the effects of interest. Treatment (natal environment provisioning and intervening patch suitability) and individual type (juvenile, male, and female) were tested as fixed terms. Interactions were tested using $F$-tests and removed if insignificant. ANOVA and t-tests were used to test for differences between individual size data. $\mathrm{R}$ (ver. 2.12.2) was used to analyse all data. Dispersal success and body size values are presented as mean $\pm \mathrm{SE}$.

\section{Results}

\section{Natal provisioning and intervening habitat suitability}

There was a significant interaction between the mites' natal environment (high vs low food) and the presence of food in the intervening habitat patch on dispersal success of the different stages (natal $\times$ inter $\times$ stage, $\mathrm{z}=-2.561$, $\mathrm{DF}=45, \mathrm{p}<0.05)$. Successful dispersal into a colonization patch of the experimental system was lower for individuals that had originated and developed in a low food natal patch and where the intervening patch was unsuitable. These low + low conditions led to the lowest successful dispersal rates for males $(73 \%)$, females $(35 \%)$, and juveniles (41\%) in comparison to the other three treatment types (Fig. 2). Individual models built to explore this interaction for each stage/gender separately revealed that food in the intervening habitat patch did not always increase dispersal success (e.g. food presence increased average dispersal in males by only $0.74 \pm 0.58$ individuals, equivalent to a change in dispersal rate of less than $2.5 \%(\mathrm{z}=1.272, \mathrm{p}=0.2$, Fig. 2), and could improve dispersal more when individuals came from a poor natal background (e.g. on average $1.1 \pm 0.3$ $(=+3.4 \%)$. More females could disperse successfully from low food natal patches when provided food in intervening patches than from high food natal patches, $\mathrm{z}=2.117$, $\mathrm{p}<0.04$, Fig. 2).

\section{Gender and development stage}

Across all treatment combinations, males showed a significantly higher dispersal success $(\mathrm{z}=3.238, \mathrm{DF}=45$, $\mathrm{p}<0.01)$ than females. Females showed a much higher dispersal success than juveniles $(73 \%$ vs $47 \%$; interaction: $\mathrm{z}=-2.530, \mathrm{DF}=45, \mathrm{p}<0.05)$ when the natal patch was low food and where the intervening patch contained food, otherwise juveniles and females dispersed at similar rates (Fig. 2).

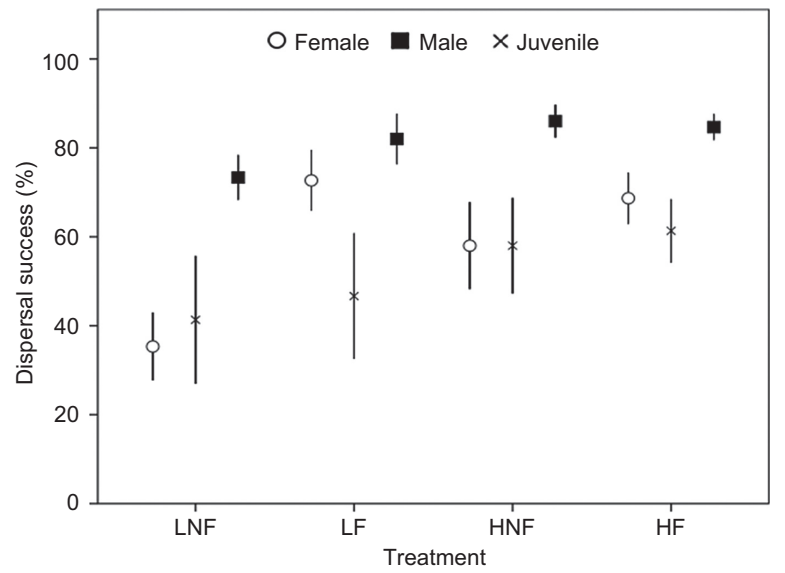

Figure 2. Effects of treatment type on the \% dispersal success (fraction of the original cohort that successfully immigrated into the colonization patch) for juveniles, adult males and females. Points are means \pm 2 SE $(n=5)$. Key: $\mathrm{H}=$ high resource (i.e. high food) natal patch environment; $\mathrm{L}=$ low resource (i.e. low food) natal patch environment; $\mathrm{F}=$ food supplied to the intervening habitat patch (suitable); NF = no food supplied to the intervening habitat patch (unsuitable). Dispersal success was only different for individuals subjected to treatment LNF where males, females and juveniles showed the lowest successful dispersal events compared to the other three treatments.

\section{Body size and interactions with intervening habitat-patch suitability}

Size at different soil mite development stages was influenced by natal patch and intervening patch provisioning (Fig. 3). A low food natal patch resulted in a significantly smaller size for eggs (high food: $0.170 \pm 0.003 \mathrm{~mm}, \mathrm{DF}=43$; low food: $0.154 \pm 0.002 \mathrm{~mm}, \mathrm{DF}=43 ; F=77.31, \mathrm{p}<0.001$ ). This trend was also found for adult males (high food: $0.639 \pm 0.01 \mathrm{~mm}, \mathrm{DF}=117$; low food: $0.586 \pm 0.009 \mathrm{~mm}$, $\mathrm{DF}=117 ; F=59.73, \mathrm{p}<0.001)$ and females (high food: $0.746 \pm 0.015 \mathrm{~mm}, \mathrm{DF}=117$; low food: $0.677 \pm 0.011$ $\mathrm{mm}, \mathrm{DF}=117 ; F=49.44, \mathrm{p}<0.001)$. However, juveniles were on average $3.6 \%$ larger in size in low food natal patches (high food: $0.301 \pm 0.007 \mathrm{~mm}, \mathrm{DF}=92$; low food: $0.312 \pm 0.005 \mathrm{~mm}, \mathrm{DF}=92 ; F=7.34, \mathrm{p}<0.01)$. This results from demographic effects: in high food natal patches good condition (large) females lay more eggs leading to a larger number of smaller juveniles competing for the same food. Unlike other stages, juveniles were also able to increase body size during dispersal when food was present (Fig. 3).

Body size was important in determining dispersal success, particularly when the intervening patch was unsuitable due to a lack of food. For the majority of treatments where the intervening patch was unsuitable, males (high food natal patch: $t=6.33, \mathrm{DF}=1, \mathrm{p}<0.05$ ), females (low food natal patch: $t=3.063, \mathrm{DF}=8, \mathrm{p}<0.01$; high food: $t=3.609, \mathrm{DF}=2, \mathrm{p}<0.05$ ), and juveniles (low food: $t=1.868, \mathrm{DF}=7, \mathrm{p}=0.05$; high food: $t=4.562, \mathrm{DF}=6$, $\mathrm{p}<0.01)$ that dispersed successfully were of significantly greater size than those that failed (Fig. 3). Males from a low food natal patch were the exception as they did not show any significant difference in size between those that dispersed 


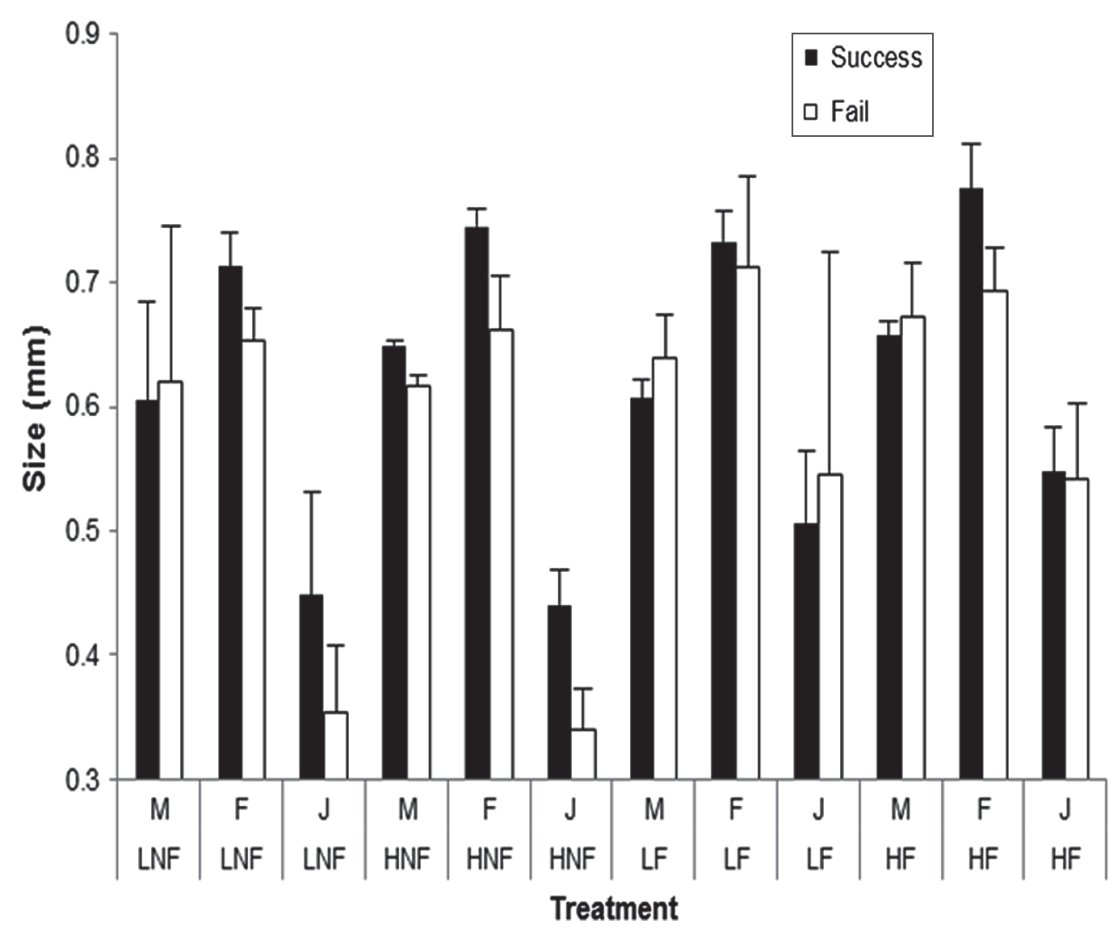

Figure 3. Bar graph showing the size $(\mathrm{mm})$ of individual male $(\mathrm{M})$, female $(\mathrm{F})$ and juvenile $(\mathrm{J})$ soil mites that dispersed successfully and those that failed to disperse for each treatment type. Bars are means \pm 2 SE. Key: $\mathrm{H}=$ high resource (i.e. high food) natal patch environment; $\mathrm{L}=$ low resource (i.e. low food) natal patch environment; $\mathrm{F}=$ food supplied to the intervening habitat patch (suitable); $\mathrm{NF}=$ no food supplied to the intervening habitat patch (unsuitable). Individuals showed a difference in size between those that dispersed successfully and those that failed when the intervening patch was unsuitable, as those that were successful tended to be larger (with the exception of males from a low food natal patch environment). F juveniles tended to be larger than NF juveniles and this growth during dispersal overrode natal patch effects.

successfully and those that failed $(t=-0.241, \mathrm{DF}=1$, $\mathrm{p}>0.05)$. When the intervening patch contained food, only females from a high food natal patch showed a significant difference in size, with those dispersing successfully being greater in size than those that failed $(t=3.253$, $\mathrm{DF}=7, \mathrm{p}<0.01)$.

\section{Discussion}

We explored how food availability in the natal patch, via its influence on individual condition, and food in the intervening habitat patches influenced the probability an individual has at successfully dispersing into a suitable colonization patch. In general, in line with expectations, a natal habitat patch with more resources was found to result in a greater probability of successful dispersal and survival of individuals. Where food was present in intervening patches, this also led to increased dispersal success in some stages from some natal backgrounds. Food provision in intervening patches never decreased dispersal.

\section{Resources in patches}

The resourcing of the intervening habitat patch had an important influence on juvenile and adult dispersal through allowing provisioning, but this was particularly important for individuals from low food natal patches. Dispersal was noticeably higher for females from low food natal patches in suitable versus unsuitable intervening patches. There was no difference in dispersal success between females from low food natal patch environments where the intervening patch was suitable and those of a high food natal patch environment. Only individuals from a low food natal patch showed lower dispersal success rates when experiencing an unsuitable intervening landscape. Thus, the spatial scale of fluctuations in resource availability clearly is a determining feature of dispersal success between patches: if individuals grow up in resource poor environments their ability to move long distance between patches with low resources is impaired. Our results demonstrate individuals can compensate for previous exposure to low food habitat when subsequently in better habitats. Resource availability within a suitable intervening patch may increase an individual's energy reserves to allow a greater physical ability to successfully disperse, or greater survival until dispersal is completed.

Mortality was difficult to measure accurately in our experiments, but our estimates were highly negatively correlated with dispersal success. Mortality did occur during dispersal and was probably due to food deprivation and/ or dehydration as individuals moved through the silicone corridors, and the energy costs involved in searching for a favourable habitat patch (Gaines and McClenaghan 1980, Baker and Rao 2004, Bowler and Benton 2009b). Generally mortality was found to be higher for individuals who had developed in a low food natal patch, regardless of life history stage. Dispersal success was significantly lower for 
these same individuals, but only where the intervening habitat patch was unsuitable. Low food natal patch environments result in reduced reproductive investment giving rise to smaller egg sizes in comparison to mothers from high food natal environments. Individuals from these smaller eggs matured into smaller adults. Therefore, individuals whose natal patch environment contained less resources may be thought of as being in a poorer condition at maturity and more likely to perish under the accumulating costs of dispersal (Benton et al. 2005, Gyllenberg et al. 2008).

By investigating the effects of natal patch resources on dispersal success and our best estimates of mortality in an environment containing intervening habitat patches we provide evidence, highlighted as crucial by recent reviews, that demonstrate how the life-history of an individual and intervening landscape suitability can influence dispersal (Travis et al. 2012). This is particularly important where there is a greater need for coupling of biological detail with theoretical models to robustly predict dispersal responses for population management purposes when environmental pressures are experienced e.g. climate change (Travis et al. 2012). Settlement behaviour in particular has been considerably understudied, not least because of the experimental challenges it poses. Our model system is simplistic in that the mites 'settle' when they have sufficient food to complete their life-cycle and there are other mites around, hence by definition, mites in the colonisation patch have 'settled' there. Travis et al. (2012) suggest that studying these behaviours (i.e. immigration and settlement) are likely to be of critical importance for understanding issues such as network connectivity, where intervening habitat patches such as stepping stones may be utilised by an individual before settling in a suitable habitat.

\section{Body size, gender and development stage}

In general, body size was shown to be an important phenotypic trait in determining individual dispersal success when the intervening landscape was unsuitable. This was regardless of whether the individual originated from a low or high food natal patch. The fact that individuals who failed to disperse tended to be of smaller size further highlights the necessity of good energy reserves or body condition to overcome the costs associated with dispersal (Barbraud et al. 2003, Warner and Shine 2008, Heidinger et al. 2010, Marini and Martins 2010, Bonte et al. 2012). Few mites remained in the source patch at the end of each experiment, across experimental combinations the majority of mites had motivation to move regardless of the presence of food in the intervening patch.

There were consistent size, gender and stage dependent differences between males, females and juveniles in how they responded to the simplistic fragmented environment. For example, males had a greater probability of successfully dispersing into a new habitat patch than juveniles and females (as per Bowler and Benton 2009b). In a species that exploits ephemeral resources, and where there is a promiscuous mating system and considerable inbreeding in local patches, males could increase fitness considerably by emigrating into new food rich patches and mating with many females. Females were consistently shown to be much larger in size than males. The larger body weight is known to benefit female fecundity once mature, but may reduce the movement ability of females, making dispersal more costly. Investment in eggs would also have reduced energy reserves available for dispersal and altered body shape that may have hindered movement (Isaacs and Byrne 1998, Miles et al. 2000). This does not contradict general findings that larger individuals disperse better in this species, as post maturation dispersal in females is not likely to be adaptive in this species. Dispersal propensity and the costs involved can vary between genders, due to different resource use and reproductive roles for each sex (Dobson 1982, Greenwood and Harvey 1982, Olsson and Shine 2003, Bonte et al. 2012) and life-history stage (Bowler 2006). Our results support the view that management of populations for any purpose should take care to identify where, and where not, interventions should be gender or developmental stage specific.

\section{Conclusion}

Continued habitat loss and fragmentation will make dispersing ability an important factor in ensuring their survival (Frankham et al. 2002). The current study suggests that the conditions experienced by an individual during development (i.e. natal/source habitat resource availability) can interact with the suitability of habitat patches during dispersal to affect the probability of successful dispersal to a more favourable habitat. This highlights that stepping stone habitats may be a useful tool in linking fragmented habitats, but only when the habitat is of suitable condition (McIntire et al. 2007). We particularly highlight the importance of suitable stepping stone habitats when natal patches contained fewer resources with dispersal increasing when suitable intervening habitat is available. This suggests we require greater investment in networks of small, suitable patches for species whose current habitat ranges are in qualitative decline. If, as we have demonstrated, species can be shown to compensate for low natal patch resources during dispersal, this will provide additional justification that investment in restoring stepping stone habitat patches is worthwhile (McIntire et al. 2007). We would argue that the biology of the at-risk species, the influence of habitat suitability on life history traits, and the condition of the current and potentially restorable habitat matrix all need to be carefully considered together when planning the restoration of highly fragmented landscapes (Collinge 2001).

Acknowledgements - The current work was completed towards the study of a Master of Research in Biodiversity and Conservation at the University of Leeds. The project proposal was developed by DOS and TGB, the experiment and analysis by DOS and TCC. All authors contributed equally to the manuscript.

\section{References}

Baker, M. B. and Rao, S. 2004. Incremental costs and benefits shape natal dispersal: theory and example with hemilepistus reaumuri. - Ecology 85: 1039-1051. 
Barbraud, C. et al. 2003. Phenotypic correlates of post-fledging dispersal in a population of greater flamingos: the importance of body condition. - J. Anim. Ecol. 72: 246-257.

Benton, T. G. and Bowler, D. E. 2012. Dispersal in invertebrates: influences on individual decisions. - In: Clobert, J. et al. (eds), Dispersal ecology and evolution. OUP.

Benton, T. G. et al. 2005. Changes in maternal investment in eggs can affect population dynamics. - Proc. R. Soc. B 272: 1351-1356.

Bodasing, M. et al. 2002. The influence of starvation on dispersal in the social spider, stegodyphus mimosarum (araneae, eresidae). - J. Arachnol. 30: 373-382.

Bonte, D. et al. 2012. Costs of dispersal. - Biol. Rev. 87: 290-312.

Bowler, D. E. 2006. Dispersal and dynamics of spatially structured populations in an experimental mite system. - Univ. of Aberdeen.

Bowler, D. E. and Benton, T. G. 2005. Causes and consequences of animal dispersal strategies: relating individual behaviour to spatial dynamics. - Biol. Rev. 80: 205-225.

Bowler, D. E. and Benton, T. G. 2009a. Impact of dispersal on population growth: the role of inter-patch distance. - Oikos 118: 403-412.

Bowler, D. E. and Benton, T. G. 2009b. Variation in dispersal mortality and dispersal propensity among individuals: the effects of age, sex and resource availability. - J. Anim. Ecol. 78: 1234-1241.

Cameron, T. C. et al. 2013. Eco-evolutionary dynamics in response to selection on life-history. - Ecol. Lett. 16: 754-763.

Clobert, J. et al. 2001. Dispersal. - Oxford Univ. Press.

Clobert, J. et al. 2012. Dispersal ecology and evolution. - Oxford Univ. Press.

Collinge, S. K. 2001. Spatial ecology and biological conservation - introduction. - Biol. Conserv. 100: 1-2.

Dobson, F. S. 1982. Competition for mates and predominant juvenile male dispersal in mammals. - Anim. Behav. 30: 1183-1192.

Einum, S. et al. 2012. Natal movement in juvenile Atlantic salmon: a body-size dependent strategy? - Popul. Ecol. 54: 285-294.

Frankham, R. et al. 2002. Introduction to conservation genetics. - Cambridge Univ. Press.

Gaines, M. S. and McClenaghan, L. R. 1980. Dispersal in small mammals. - Annu. Rev. Ecol. Syst. 11: 163-196.

Greenwood, P. J. and Harvey, P. H. 1982. The natal and breeding dispersal of birds. - Annu. Rev. Ecol. Syst. 13: 1-21.

Gyllenberg, M. et al. 2008. Evolution of condition-dependent dispersal under kin competition. - J. Math. Biol. 57: 285-307.
Heidinger, I. M. M. et al. 2010. Patch connectivity and sand dynamics affect dispersal-related morphology of the bluewinged grasshopper oedipoda caerulescens in coastal grey dunes. - Insect Conserv. Divers. 3: 205-212.

Isaacs, R. and Byrne, D. N. 1998. Aerial distribution, flight behaviour and eggload: their inter-relationship during dispersal by the sweetpotato whitefly. - J. Anim. Ecol. 67: 741-750.

Loe, L. E. et al. 2010. No evidence of juvenile body mass affecting dispersal in male red deer. - J. Zool. 280: 84-91.

Marini, O. J. and Martins, R. P. 2010. Nymphalid butterfly dispersal among forest fragments at Serra da Canastra National park, Brazil. - J. Insect Conserv. 14: 401-411.

McCauley, S. J. 2010. Body size and social dominance influence breeding dispersal in male Pachydiplax longipennis (Odonata). - Ecol. Entolmol. 35: 377-385.

McIntire, E. J. B. et al. 2007. Designing a network for butterfly habitat restoration: where individuals, populations and landscapes interact. - J. Appl. Ecol. 44: 725-736.

Miles, D. B. et al. 2000. Reproductive burden, locomotor performance, and the cost of reproduction in free ranging lizards. - Evolution 54: 1386-1395.

Olsson, M. and Shine, R. 2003. Female-biased natal and breeding dispersal in an alpine lizard, niveoscincus microlepidotus. - Biol. J. Linn. Soc. 79: 277-283.

Plaistow, S. J. et al. 2004. Age and size at maturity: sex, environmental variability and developmental thresholds. - Proc. R. Soc. B 271: 919-924.

Telfer, S. et al. 2003. Parentage assignment detects frequent and large-scale dispersal in water voles. - Mol. Ecol. 12: 1939-1949.

Thomas, C. D. 2000. Dispersal and extinction in fragmented landscapes. - Proc. R. Soc. B 267: 139-145.

Travis, J. M. J. et al. 2012. Modelling dispersal: an eco-evolutionary framework incorporating emigration, movement, settlement behaviour and the multiple costs involved. - Meth. Ecol. Evol. 3: 628-641.

van Vuren, D. and Armitage, K. B. 1994. Survival of dispersing and philopatric yellow-bellied marmots - what is the cost of dispersal. - Oikos 69: 179-181.

Warner, D. A. and Shine, R. 2008. Determinants of dispersal distance in free-ranging juvenile lizards. - Ethology 114: 361-368.

Waser, P. M. et al. 1994. Death and disappearance - estimating mortality risks associated with philopatry and dispersal. - Behav. Ecol. 5: 135-141. 